\title{
Brain Perfusion in Corticobasal Syndrome with Progressive Aphasia
}

\author{
Yoshitake Abe Noriyuki Kimura Megumi Goto Yasuhiro Aso \\ Etsuro Matsubara \\ Department of Neurology, Faculty of Medicine, Oita University, Yufu, Japan
}

\section{Key Words}

Corticobasal syndrome $\cdot$ Aphasia $\cdot$ Brain perfusion SPECT $\cdot$ Statistical Parametric Mapping 8

\begin{abstract}
Background: Brain perfusion may differ between patients with corticobasal syndrome (CBS) with and without aphasia. Methods: Twenty-six ( 9 males and 17 females; mean age $76 \pm 5.3$ years) patients with CBS were enrolled in the study. Brain MRI and single-photon emission computed tomography were performed in all subjects. Language was evaluated using the Standard Language Test of Aphasia. The patients were divided into two subgroups according to the presence or absence of progressive aphasia. Differences in the regional cerebral blood flow ( $\mathrm{rCBF}$ ) between the two groups were detected based on voxel-by-voxel group analysis using Statistical Parametric Mapping 8. Results: All patients exhibited asymmetric motor symptoms and signs, including limb apraxia, bradykinesia, and akinetic rigidity. Of 26 patients, 9 had a clinically obvious language disturbance, characterized as nonfluent aphasia. Almost all CBS patients with aphasia exhibited cortical atrophy predominantly in the left frontal and temporal lobes with widening of the Sylvian fissure on MRI. The rCBF in the left middle frontal gyrus differed significantly between CBS patients with and without aphasia. Conclusion: CBS patients with aphasia exhibit motor symptoms predominantly on the right side and cortical atrophy mainly in the left perisylvian cortices. In particular, left frontal dysfunction might be related to nonfluent aphasia in CBS.

\section{Introduction}

Corticobasal degeneration (CBD) is a neurodegenerative disorder characterized by progressive asymmetric rigidity and apraxia with other variable findings, including cortical sensory loss, alien limb phenomena, myoclonus, and dystonia [1]. Patients with pathologi- 
cally confirmed CBD often present with various cognitive deficits during the course of the disease [2-4]. Pathologic heterogeneity is also reported in clinically diagnosed CBD patients [5-7]. Therefore, recent studies use the term corticobasal syndrome (CBS) for the clinical syndrome, and the term CBD for the histopathologic disorder [7]. The cognitive deficits of CBS include language disturbances and frontotemporal dysfunction. Language disturbance is particularly focused on as the diagnostic criterion for CBS because over one third of all CBS patients have aphasia, characterized as the nonfluent type [8-10]. A few studies have evaluated the relationship between cognitive deficits and MRI findings in CBD [11-13]. Frattali et al. [11] suggest that aphasia in CBD is associated primarily with left frontal and parietal cortical damage, and subcortical white matter and corpus callosum abnormalities.

Brain perfusion single-photon emission computed tomography (SPECT) imaging is widely available and provides an indirect marker of neuronal function. Characteristic perfusion SPECT findings of CBS are the presence of asymmetric perfusion and a decreased regional cerebral blood flow ( $\mathrm{rCBF}$ ) in the frontoparietal cortices, basal ganglia, thalamus, pons, and cerebellum $[14,15]$. To our knowledge, there are few studies of the relationship between language disturbances and perfusion SPECT in CBS. It is important to confirm the SPECT patterns of CBS with and without aphasia for an accurate diagnosis and to gain a better understanding of the distribution of pathologic changes in CBS. The aim of the present study was to elucidate the differences in the SPECT pattern of CBS patients with and without aphasia, using the Statistical Parametric Mapping 8 (SPM8) program.

\section{Materials and Methods}

\section{Subjects}

We consecutively enrolled 26 patients with CBS ( 9 males and 17 females; mean age 76 \pm 5.3 years) who were admitted to the Department of Neurology, Oita University Hospital, between 2006 and 2014. CBS was diagnosed according to the diagnostic criteria proposed by Mathew et al. [9]. All patients satisfied the clinical criteria for possible CBD. Mean $( \pm$ SD) disease duration was $2.3 \pm 1.2$ years, ranging from 1 to 5 years. All patients were righthanded and underwent a clinical evaluation that included past medical history as well as physical and neurologic examination. Information regarding age, sex, and disease duration was extracted from the medical records. Language was evaluated using the Standard Language Test of Aphasia [16]. The patients were divided into two subgroups according to the presence or absence of aphasia. Brain MRI and brain perfusion SPECT were performed for diagnosis. All patients were examined using T1-weighted, T2-weighted, and fluid-attenuated inversion recovery images on a 1.5-tesla scanner (Excelart Vantage; Toshiba Medical System Corp., Tokyo, Japan). Axial and coronal MRIs were obtained and retrospectively reviewed by two registered neurologists blinded to the medical information and used for diagnosis of CBS. Brain atrophy and abnormal signal intensity were examined visually and qualitatively in the cerebral cortex, white matter, and basal ganglia. In case of disagreement, they reviewed the images again together to reach a consensus. We also studied 26 age- and sex-matched control subjects ( 8 males and 18 females; mean age $76.6 \pm 5.8$ years) who had no neurologic disorders or cognitive impairment for a voxel-by-voxel group analysis using SPM8.

Between-group comparisons of CBS patients with and without aphasia and controls were performed with the $\chi^{2}$ test for sex distribution and a Kruskal-Wallis test, followed by a MannWhitney $U$ test for age at examination and mean global CBF. The comparison of disease duration was performed with a Mann-Whitney U test between CBS patients with and without aphasia. A p value $<0.05$ was considered statistically significant. 


\section{Brain SPECT Imaging}

The patients were asked to assume a comfortable supine position with the eyes closed in a quiet surrounding. After intravenous injection of $99 \mathrm{mTc}$ ethyl cysteinate dimer $(600 \mathrm{MBq}$; FUJIFILM RI Pharma Co., Ltd., Chiba, Japan), its passage to the brain was monitored using a rectangular large-field gamma camera (E. Cam Signature; Toshiba Medical System Corp., Tochigi, Japan). The Patlak plot method was used to measure the mean global CBF on $99 \mathrm{mTc}$ ethyl cysteinate dimer cerebral blood perfusion SPECT [17]. Data comprising a sequence of 120 frames were acquired at a rate of $1 \mathrm{~Hz}$ with a $128 \times 128$ matrix. Ten minutes after the angiography, SPECT images were obtained using a rotating dual-head gamma camera equipped with low-energy, high-resolution, and parallel-hole collimators. Energy windows were set at $140 \mathrm{keV} \pm 20 \%$, and 90 views were obtained throughout $360^{\circ}$ of rotation $(128 \times$ 128 matrix, $1.95 \mathrm{~mm} /$ pixel), with an acquisition time of $140 \mathrm{~s} /$ cycle repeated 6 times. Data were processed using a GMS-7700A/EI (Toshiba Medical System Corp.). All images were reconstructed using a ramp-filtered back projection and then three-dimensionally smoothed with a Butterworth filter (order 8, cutoff 0.25 cycles/pixel). The reconstructed images were corrected for gamma ray attenuation using the Chang method $(\mu=0.09)$.

\section{SPECT Image Analysis Using SPM8}

A voxel-by-voxel group study was performed using SPM8 (Wellcome Department of Cognitive Neurology, University College, London, UK), running on MATLAB version R2009b (The MathWorks, Inc., Natick, Mass., USA). The images were initially converted from DICOM files to the analysis format using MRIcro and transferred to SPM8. The mean pixel intensity across all slices of the imaging volume was calculated. The pixel threshold was set to $80 \%$ of the whole-brain mean to eliminate background noise and partial volume effects at the edge of the brain. The data were standardized with the Montreal Neurological Institute atlas using a 12-parameter affine transformation, followed by nonlinear transformations and a trilinear interpolation. The final image format was 16 bit, with a size of $79 \times 95 \times 69$ voxels and a voxel size of $2 \times 2 \times 2 \mathrm{~mm}$. The standardized data were smoothed by a Gaussian filter (full width at half maximum: $12 \mathrm{~mm}$ ). Regional CBF differences were assessed as an intergroup comparison using the 'compare-populations: $1 \mathrm{scan} /$ subject (two-sample t test)'. Each individual global count was normalized by proportional scaling. The resulting set of values for each comparison constituted a statistical parametric map of the $t$ statistic SPM $\{\mathrm{t}\}$. The SPM $\{\mathrm{t}\}$ maps were then transformed to the unit of normal distribution (SPM $\{z\}$ ). The SPM $\{t\}$ maps were initially obtained at a height threshold of $\mathrm{p}<0.001$ (uncorrected), with an extent threshold of 50 voxels. Montreal Neurological Institute coordinates were finally converted to Talairach brain coordinates.

\section{Statement of Ethics}

Fully informed consent was obtained, and all patients and controls agreed to participate in this study.

\section{Results}

Table 1 summarizes the clinical and demographic characteristics of CBS patients with and without aphasia. Of 26 patients, 9 exhibited clinically obvious language disturbances, such as speech hesitancy, distortions, phonemic errors, and handwriting difficulty. Comprehension and object knowledge were relatively preserved. These language disturbances are characterized as nonfluent aphasia. The subgroups did not significantly differ in age at examination, sex distribution, or disease duration. Although the mean global CBF value tended to 
Table 1. Summary of the clinical and demographic characteristics of patients with CBS

\begin{tabular}{lcccl}
\hline & $\begin{array}{l}\text { CBS with aphasia } \\
(\mathrm{n}=9)\end{array}$ & $\begin{array}{l}\text { CBS without aphasia } \\
(\mathrm{n}=17)\end{array}$ & $\begin{array}{l}\text { Controls } \\
(\mathrm{n}=26)\end{array}$ & $\mathrm{p}$ \\
\hline Age, years & $75.9 \pm 5.7$ & $76.1 \pm 5.3$ & $76.6 \pm 5.8$ & 0.98 \\
Sex, M/F & $3 / 6$ & $6 / 11$ & $8 / 18$ & 0.988 \\
Disease duration, years & $2.4 \pm 1.3$ & $2.2 \pm 1.1$ & - & 0.71 \\
CBF, ml/100 g/min & $36.0 \pm 2.6$ & $38.4 \pm 3.7$ & $40.8 \pm 4.4$ & 0.007 \\
\hline
\end{tabular}

Values are expressed as means \pm SD or numbers. $\mathrm{p}<0.05$ was considered statistically significant.

Table 2. Summary of the motor impairment and brain MRI findings in CBS patients with and without aphasia

\begin{tabular}{|c|c|c|c|c|c|c|c|}
\hline \multirow[t]{2}{*}{ Patient } & \multirow{2}{*}{$\begin{array}{l}\text { Duration, } \\
\text { years }\end{array}$} & \multirow[t]{2}{*}{ Aphasia } & \multicolumn{3}{|c|}{ Motor impairment } & \multicolumn{2}{|c|}{ Brain atrophy on MRI } \\
\hline & & & side & extrapyramidal & apraxia & side & location \\
\hline 1 & 1 & NF & $\mathrm{R}$ & + & + & $\mathrm{L}$ & $\mathrm{F}, \mathrm{T}, \mathrm{P}$ \\
\hline 2 & 4 & $\mathrm{NF}$ & $\mathrm{R}$ & + & + & $\mathrm{L}$ & $\mathrm{F}, \mathrm{P}$ \\
\hline 3 & 5 & $\mathrm{NF}$ & $\mathrm{R}$ & + & + & $\mathrm{L}$ & $\mathrm{F}, \mathrm{T}, \mathrm{P}$ \\
\hline 4 & 3 & $\mathrm{NF}$ & $\mathrm{R}$ & + & + & $\mathrm{L}$ & $\mathrm{F}, \mathrm{T}, \mathrm{P}$ \\
\hline 5 & 2 & $\mathrm{NF}$ & $\mathrm{R}$ & + & + & $\mathrm{L}$ & $\mathrm{F}, \mathrm{T}, \mathrm{P}$ \\
\hline 6 & 1.5 & $\mathrm{NF}$ & $\mathrm{R}$ & + & + & $\mathrm{L}$ & $\mathrm{F}, \mathrm{P}, \mathrm{O}$ \\
\hline 7 & 1.5 & $\mathrm{NF}$ & $\mathrm{R}$ & + & + & $\mathrm{L}$ & $\mathrm{F}, \mathrm{T}, \mathrm{P}$ \\
\hline 8 & 2 & $\mathrm{NF}$ & $\mathrm{L}$ & + & - & $\mathrm{R}$ & $\mathrm{F}, \mathrm{T}, \mathrm{P}$ \\
\hline 9 & 2 & NF & $\mathrm{R}$ & + & - & $\mathrm{L}$ & $\mathrm{F}, \mathrm{P}$ \\
\hline 10 & 1.3 & - & $\mathrm{R}$ & + & + & $\mathrm{L}$ & $\mathrm{F}, \mathrm{P}$ \\
\hline 11 & 2 & - & $\mathrm{L}$ & + & + & B & $\mathrm{F}, \mathrm{P}$ \\
\hline 12 & 1.3 & - & $\mathrm{R}$ & + & + & $\mathrm{L}$ & $\mathrm{F}, \mathrm{P}$ \\
\hline 13 & 3 & - & $\mathrm{L}$ & + & + & $\mathrm{R}$ & $\mathrm{P}$ \\
\hline 14 & 3.5 & - & $\mathrm{L}$ & + & - & $\mathrm{R}$ & $\mathrm{P}$ \\
\hline 15 & 3 & - & $\mathrm{L}$ & + & + & $\mathrm{R}$ & $\mathrm{F}, \mathrm{T}, \mathrm{P}$ \\
\hline 16 & 2 & - & $\mathrm{L}$ & + & + & $\mathrm{R}$ & $\mathrm{F}, \mathrm{T}, \mathrm{P}$ \\
\hline 17 & 5 & - & $\mathrm{R}$ & + & - & $\mathrm{L}$ & $\mathrm{F}, \mathrm{T}, \mathrm{P}, \mathrm{O}$ \\
\hline 18 & 2 & - & $\mathrm{R}$ & + & - & $\mathrm{L}$ & $\mathrm{F}, \mathrm{T}, \mathrm{P}$ \\
\hline 19 & 1 & - & $\mathrm{L}$ & + & - & B & $\mathrm{F}, \mathrm{P}$ \\
\hline 20 & 3 & - & $\mathrm{L}$ & + & - & $\mathrm{R}$ & $\mathrm{F}, \mathrm{P}$ \\
\hline 21 & 3 & - & $\mathrm{R}$ & + & + & $\mathrm{L}$ & $\mathrm{F}, \mathrm{P}$ \\
\hline 22 & 2.5 & - & $\mathrm{R}$ & + & + & $\mathrm{L}$ & $\mathrm{F}, \mathrm{T}, \mathrm{P}$ \\
\hline 23 & 1 & - & $\mathrm{L}$ & + & + & $\mathrm{R}$ & $\mathrm{F}, \mathrm{P}$ \\
\hline 24 & 2 & - & $\mathrm{R}$ & + & + & $\mathrm{L}$ & $\mathrm{F}, \mathrm{T}, \mathrm{P}$ \\
\hline 25 & 1 & - & $\mathrm{R}$ & + & + & $\mathrm{L}$ & $\mathrm{F}, \mathrm{T}, \mathrm{P}$ \\
\hline 26 & 1 & - & $\mathrm{R}$ & + & + & $\mathrm{L}$ & $\mathrm{F}, \mathrm{T}, \mathrm{P}$ \\
\hline
\end{tabular}

$\mathrm{NF}=$ Nonfluent aphasia; side = more affected side; extrapyramidal = extrapyramidal signs; $\mathrm{R}=$ right; $\mathrm{L}=$ left; $\mathrm{B}=$ bilateral; $\mathrm{F}=$ frontal lobe; $\mathrm{T}$ = temporal lobe; $\mathrm{P}=$ parietal lobe; $\mathrm{O}=$ occipital lobe.

be lower in the CBS-with-aphasia group than in the CBS-without-aphasia group, the difference was not statistically significant $(\mathrm{p}<0.06)$. All patients exhibited asymmetric motor symptoms and signs, including limb apraxia, bradykinesia, and akinetic rigidity (table 2). Levodopa treatment was not effective for the parkinsonian symptoms. Of the total of 26 patients, 17 exhibited predominantly right-sided symptoms, and the remaining 9 patients exhibited predominantly left-sided symptoms. Moreover, 8 of the 9 CBS patients with aphasia exhibited 

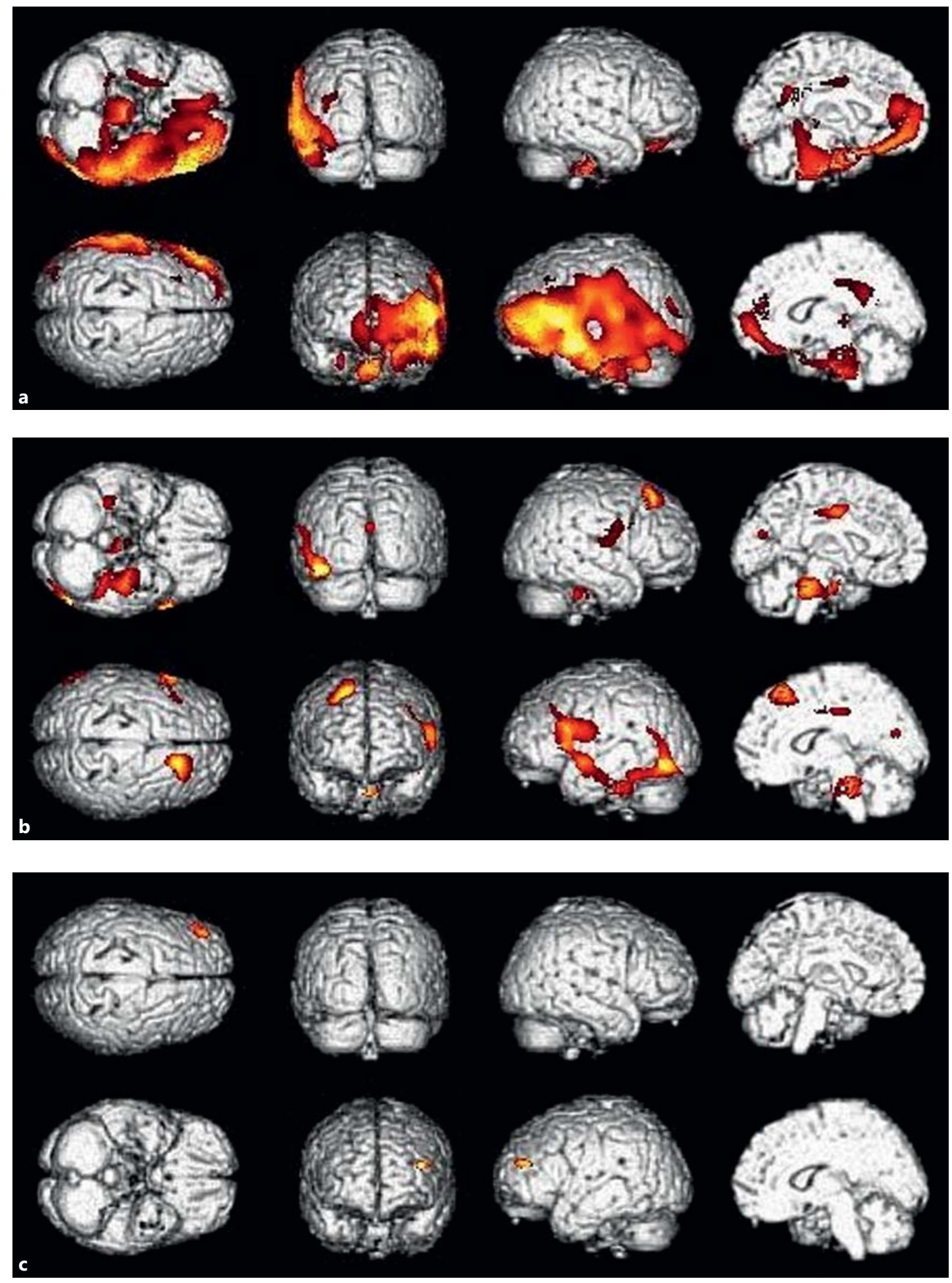

Fig. 1. SPM brain map of the group comparison. a Group comparison between the CBS-with-aphasia group and controls. Cerebral perfusion was significantly decreased mainly in the left frontal, temporal, and occipital cortices adjacent to the Sylvian fissure in CBS patients with aphasia. b Group comparison between the CBS-without-aphasia group and controls. Cerebral perfusion was significantly decreased mainly in the left frontal, temporal, and occipital cortices, as well as the cerebellum and pons in CBS patients without aphasia. c Group comparison between CBS patients with and without aphasia. Cerebral perfusion was significantly decreased in the left middle frontal gyrus in CBS patients with aphasia. 
Table 3. Locations and peaks of significantly decreased rCBF in CBS patients with and without aphasia as well as controls

\begin{tabular}{|c|c|c|c|c|c|c|}
\hline & \multirow{2}{*}{$\begin{array}{l}\text { Voxel } \\
\text { level }\{Z\}\end{array}$} & \multirow{2}{*}{$\begin{array}{l}\text { Voxel } \\
\text { p (unc.) }\end{array}$} & \multicolumn{3}{|c|}{ Talairach coordinates } & \multirow[t]{2}{*}{ Region } \\
\hline & & & $\mathrm{x}$ & $\mathrm{y}$ & $\mathrm{z}$ & \\
\hline \multirow{11}{*}{$\begin{array}{l}\text { CBS with aphasia } \\
\text { vs. controls }\end{array}$} & 5.61 & 0.000 & -47.52 & 13.66 & 1.16 & L insula \\
\hline & 5.40 & 0.000 & -41.58 & -0.84 & -16.78 & L superior temporal gyrus \\
\hline & 4.98 & 0.000 & -37.62 & -22.68 & -25.78 & L parahippocampal gyrus \\
\hline & 3.79 & 0.000 & 7.92 & -41.43 & 26.03 & $\mathrm{R}$ posterior cingulate gyrus \\
\hline & 3.65 & 0.000 & 13.86 & -25.47 & 34.44 & $\mathrm{R}$ cingulate gyrus \\
\hline & 3.67 & 0.000 & 29.70 & -17.01 & -29.43 & R uncus \\
\hline & 3.54 & 0.000 & -11.88 & -6.00 & 35.31 & L cingulate gyrus \\
\hline & 3.39 & 0.000 & 31.68 & -36.56 & -31.81 & R cerebellum \\
\hline & 3.33 & 0.000 & 3.96 & -28.97 & 3.29 & $\mathrm{R}$ thalamus \\
\hline & 3.33 & 0.000 & -9.90 & -51.90 & 30.23 & L precuneus \\
\hline & 3.22 & 0.001 & -33.66 & -80.55 & 20.61 & L middle occipital gyrus \\
\hline \multirow{10}{*}{$\begin{array}{l}\text { CBS without aphasia } \\
\text { vs. controls }\end{array}$} & 4.36 & 0.000 & 17.82 & 29.70 & 50.10 & R superior frontal gyrus \\
\hline & 3.95 & 0.000 & 13.86 & -21.60 & 34.24 & $\mathrm{R}$ cingulate gyrus \\
\hline & 3.90 & 0.000 & 0.00 & -34.12 & -21.84 & L cerebellum \\
\hline & 3.18 & 0.001 & 0.00 & -18.87 & -27.65 & Pons \\
\hline & 3.78 & 0.000 & -57.42 & 18.27 & 15.67 & L inferior frontal gyrus \\
\hline & 3.69 & 0.000 & -35.64 & -26.45 & -23.91 & L parahippocampal gyrus \\
\hline & 3.65 & 0.000 & -15.84 & -21.50 & 36.08 & $\mathrm{~L}$ cingulate gyrus \\
\hline & 3.55 & 0.000 & 43.56 & -7.01 & 15.09 & $\mathrm{R}$ insula \\
\hline & 3.35 & 0.000 & 1.98 & -70.77 & 21.97 & R cuneus \\
\hline & 3.31 & 0.000 & 35.64 & -34.37 & -26.88 & R cerebellum \\
\hline $\begin{array}{l}\text { CBS with aphasia vs. } \\
\text { CBS without aphasia }\end{array}$ & 3.25 & 0.001 & -37.62 & 45.58 & 17.98 & L middle frontal gyrus \\
\hline
\end{tabular}

unc. $=$ Uncorrected; $\mathrm{R}=$ right; $\mathrm{L}=$ left.

predominantly right-sided motor symptoms. Brain MRI in the CBS patients showed bilateral, usually asymmetric, cortical atrophy, mainly in the frontal and parietal cortices. In some patients, the lesions extended to the temporal or occipital cortices. Asymmetric cortical atrophy was more severe on the side contralateral to the clinically more affected side in 24 patients. The atrophy was equivalent in both hemispheres in the remaining 2 patients. Almost all CBS patients with aphasia had cortical atrophy predominantly in the left frontal and temporal lobes adjacent to the Sylvian fissure as well as in the parietal lobe. There was no abnormal signal intensity in the cortex, white matter, and basal ganglia. Figure 1 shows the comparison of the absolute rCBF between CBS patients with and without aphasia and controls using SPM8. SPM8 analysis revealed asymmetric perfusion and a decreased rCBF predominantly in the left cerebral hemisphere in both CBS groups compared to controls. The CBSwith-aphasia group had a significantly decreased rCBF in the insula, superior temporal gyrus, parahippocampal gyrus, precuneus, and middle occipital gyrus of the left hemisphere and in the posterior cingulate gyrus, uncus, cerebellum, and thalamus of the right hemisphere as well as the bilateral cingulate gyri, compared to controls (fig. 1a, table 3). CBS patients without aphasia had a significantly decreased $\mathrm{rCBF}$ in the inferior frontal gyrus and parahippocampal gyrus of the left hemisphere and the superior frontal gyrus, insula, and cuneus of the right hemisphere, as well as the bilateral cingulate gyrus and cerebellum, compared to controls (fig. 1b, table 3). Moreover, a significant difference in the rCBF in the left middle frontal gyrus was detected between CBS patients with and without aphasia (fig. 1c, table 3). 


\section{Discussion}

The present study examined the differences in the rCBF between CBS patients with and without aphasia using the SPM8 program. Compared with controls, CBS patients with and without aphasia showed asymmetric perfusion and a decreased $\mathrm{rCBF}$ in the extended cerebral cortex, cerebellum, and brainstem regions. The hypoperfusion area in CBS patients with aphasia was more severe and extensive in the left cerebral cortex than that in CBS patients without aphasia. Moreover, direct comparison between CBS patients with and without aphasia revealed a significant difference in the rCBF in the left frontal cortex.

Most CBS patients present with cognitive impairment, including language disturbances, during the course of the disease or even at onset [6-9]. In the present study, all patients exhibited asymmetric extrapyramidal signs and/or apraxia, and 9 of 26 patients (34.6\%) had a language disturbance characterized as nonfluent aphasia. The mean disease duration in our patients upon admission to our hospital was $2.3 \pm 1.2$ years. These findings reconfirmed that aphasia, particularly the nonfluent type, is more frequent in CBS patients than previously considered and a prominent characteristic. Moreover, 8 of 9 CBS patients with aphasia exhibited predominantly right-sided motor symptoms. A previous study of CBD with aphasia showed predominant motor impairment on the right side in 4 of 8 patients. In the other 4 patients, however, bilateral involvement was evidenced by clinical and MRI findings [11]. Therefore, language disturbances in CBS patients tend to be associated with involvement of the left hemisphere.

Characteristic MRI findings of patients with CBS included asymmetric cortical atrophy in the frontal and parietal lobes and basal ganglia $[12,13]$. The cortical atrophy in these regions was more severe on the side contralateral to the clinically more affected side. Similarly, most of our CBS patients had asymmetric cortical atrophy mainly in the frontal, temporal, and parietal lobes on the side contralateral to the clinically more affected side, whereas 2 patients showed equivalent atrophy in both hemispheres. Moreover, CBS patients with aphasia had cortical atrophy mainly in the left frontal, temporal, and parietal cortices, and only 1 patient had cortical atrophy bilaterally but predominantly in the right hemisphere. These results are consistent with those of a previous study, suggesting a relationship between aphasia and left frontal and parietal cortical involvement in $\operatorname{CBD}[3,10,11,13]$.

Characteristic perfusion SPECT findings of CBS are the presence of asymmetric perfusion and a decreased $\mathrm{rCBF}$ in the frontoparietal cortices, basal ganglia, thalamus, pons, and cerebellum $[14,15]$. There are, however, few studies of the relationship between language disturbances and perfusion SPECT in CBS. A single case report showed hypoperfusion in the frontal and temporal cortices, the insula, and the parietal lobe, including the parietal lobules and precuneus, in CBS patients with aphasia [18]. In the present study, we used the absolute rCBF data to evaluate cerebral perfusion using SPM8 analysis according to a previous study, indicating that the adjusted $\mathrm{rCBF}$ data may not be suitable for analyzing the rCBF in CBS [12]. SPM8 analysis revealed asymmetric perfusion and a decreased rCBF predominantly in the left cerebral hemisphere, including the frontal, temporal, and occipital cortices, and the thalamus, cerebellum, and pons in patients with CBS with or without aphasia compared to controls. This predominance of brain dysfunction may account for the high frequency of patients with a clinically more affected right side. These findings are consistent with the characteristic SPECT pattern of CBS. Moreover, CBS patients with aphasia showed more severe and extended hypoperfusion, mainly in the left frontal and temporal cortices adjacent to the Sylvian fissure. A previous positron emission tomography study suggested that non-fluent aphasia was associated with the anterior perisylvian region, whereas others described that the language function, particularly phonologic processing, is related to the inferior frontal, superior temporal, and supramarginal gyri, as well as the anterior insula of the left hemisphere [10, 
$17,19]$. In the present SPECT study, the rCBF in CBS patients with aphasia was significantly decreased in the left middle frontal gyrus compared to that in CBS patients without aphasia, despite the lack of a significant difference in disease duration and global cerebral perfusion. These findings therefore suggest that more extensive brain dysfunction, particularly in left frontal regions, might be related to nonfluent aphasia in CBS.

The present study has several limitations. First, patients were diagnosed based solely on clinical signs and symptoms without pathologic confirmation. Second, the distinct SPECT patterns in CBS patients with and without aphasia must be considered preliminary because of the relatively small number of CBS patients with aphasia. Further studies with larger samples are needed to confirm our results.

In conclusion, nonfluent aphasia in patients with CBS is more common than previously considered and an important finding for the diagnosis of CBS. CBS patients with aphasia exhibited predominantly right-sided motor symptoms and cortical atrophy mainly in the left perisylvian cortices. In particular, the left frontal dysfunction might be related to nonfluent aphasia in patients with CBS.

\section{Disclosure Statement}

Yoshitake Abe, Noriyuki Kimura, Megumi Goto, Yasuhiro Aso, and Etsuro Matsubara declare that they have no conflicts of interest to declare.

\section{References}

1 Rebeiz JJ, Kolodny EH, Richardson EP Jr: Corticodentatonigral degeneration with neuronal achromasia. Arch Neurol 1968;18:20-33.

-2 Grimes DA, Lang AE, Bergeron CB: Dementia as the most common presentation of cortical-basal ganglionic degeneration. Neurology 1999;53:1969-1974.

-3 Murray R, Neumann M, Forman MS, Farmer J, Massimo L, Rice A, Miller BL, Johnson JK, Clark CM, Hurtig HI, Gorno-Tempini ML, Lee VM, Trojanowski JQ, Grossman M: Cognitive and motor assessment in autopsy-proven corticobasal degeneration. Neurology 2007;68:1274-1283.

-4 Kimura N, Kumamoto T, Hanaoka T, Hazama Y, Nakamura K, Arakawa R: Corticobasal degeneration presenting with progressive conduction aphasia. J Neurol Sci 2008;269:163-168.

5 Schneider JA, Watts RL, Gearing M, Brewer RP, Mirra SS: Corticobasal degeneration: neuropathologic and clinical heterogeneity. Neurology 1997;48:959-969.

6 Kertesz A, Martinez-Lage P, Davidson W, Munoz DG: The corticobasal degeneration syndrome overlaps progressive aphasia and frontotemporal dementia. Neurology 2000;55:1368-1375.

7 Boeve BF: The multiple phenotypes of corticobasal syndrome and corticobasal degeneration: implications for further study. J Mol Neurosci 2011;45:350-353.

8 McMonagle P, Blair M, Kertesz A: Corticobasal degeneration and progressive aphasia. Neurology 2006;67: 1444-1451.

9 Mathew R, Bak TH, Hodges JR: Diagnostic criteria for corticobasal syndrome: a comparative study. J Neurol Neurosurg Psychiatry 2012;83:405-410.

10 Graham NL, Bak T, Patterson K, Hodges JR: Language function and dysfunction in corticobasal degeneration. Neurology 2003;61:493-499.

-11 Frattali CM, Grafman J, Patronas N, Makhlouf F, Litvan I: Language disturbances in corticobasal degeneration. Neurology 2000;54:990-992.

12 Jutten K, Pieperhoff P, Sudmeyer M, Schleicher A, Ferrea S, Caspers S, Zilles K, Schnitzler A, Amunts K, Lux S: Neuropsychological and brain volume differences in patients with left- and right-beginning corticobasal syndrome. PLoS One 2014;9:e110326.

13 Lee SE, Rabinovici GD, Mayo MC, Wilson SM, Seeley WW, DeArmond SJ, Huang EJ, Trojanowski JQ, Growdon ME, Jang JY, Sidhu M, See TM, Karydas AM, Gorno-Tempini ML, Boxer AL, Weiner MW, Geschwind MD, Rankin KP, Miller BL: Clinicopathological correlations in corticobasal degeneration. Ann Neurol 2011;70:327-340.

$\checkmark 14$ Hossain AK, Murata Y, Zhang L, Taura S, Saitoh Y, Mizusawa H, Oda K, Matsushima E, Okubo Y, Shibuya H: Brain perfusion SPECT in patients with corticobasal degeneration: analysis using statistical parametric mapping. Mov Disord 2003;18:697-703. 
15 Ukmar M, Moretti R, Torre P, Antonello RM, Longo R, Bava A: Corticobasal degeneration: structural and functional MRI and single-photon emission computed tomography. Neuroradiology 2003;45:708-712.

16 Mimura M, Kato M, Kato M, Sano Y, Kojima T, Naeser M, Kashima H: Prospective and retrospective studies of recovery in aphasia. Changes in cerebral blood flow and language functions. Brain 1998;121:2083-2094.

17 Zatorre RJ, Meyer E, Gjedde A, Evans AC: PET studies of phonetic processing of speech: review, replication, and reanalysis. Cereb Cortex 1996;6:21-30.

18 Caso F, Onofrio F, Falautano M, Todeschini P, Migliaccio R, Comi G, Perani D, Magnani G: From primary progressive aphasia to corticobasal syndrome: two clinical and rCBF functional reports. Neurocase 2013;19: 201-207.

19 Demonet JF, Fiez JA, Paulesu E, Petersen SE, Zatorre RJ: PET studies of phonological processing: a critical reply to Poeppel. Brain Lang 1996;55:352-379. 